\title{
Convergencia digital y software libre en el EEES: algunas experiencias con Gretl ${ }^{\star}$
}

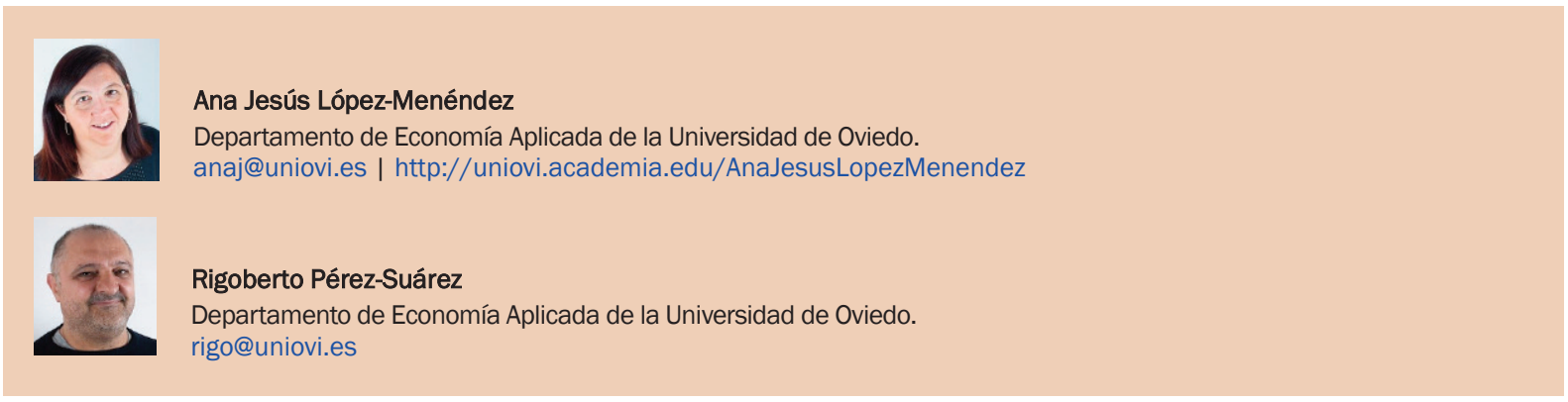

|Fecha presentación: 04/07/2011 | Aceptación: 21/06/2012 |Publicación: 20/12/2012

\begin{abstract}
Resumen
La utilización de software libre y abierto (Free Open Source Software: FOSS) aporta numerosas ventajas en el ámbito universitario, que resultan aún más patentes en el marco del Espacio Europeo de Educación Superior (EEES). Las principales características de FOSS (libertad de uso de programas, acceso al código fuente, estudio y adaptación, distribución de copias e implementación de mejoras que beneficien a toda la comunidad) aparecen relacionadas con competencias como creatividad, trabajo en equipo o adaptación a nuevas situaciones. Este trabajo examina el papel del software libre en el ámbito universitario y su potencial para reducir la brecha digital, presentando también algunas experiencias recientes.
\end{abstract}

Palabras clave: FOSS, Espacio Europeo de Educación Superior (EEES), Moodle, Open Course Ware (OCW), Gretl

\begin{abstract}
Resum
La utilització de programari lliure i obert (Free Open Source Software: FOSS) aporta nombrosos avantatges en l'àmbit universitari, que resulten encara més patents en el marc de l'Espai Europeu d'Educació Superior (EEES). Les principals característiques del FOSS (llibertat d'ús de programes, accés al codi font, estudi i adaptació, distribució de còpies i implementació de millores que beneficien a tota la comunitat) apareixen relacionades amb competències com a creativitat, treball en equip o adaptació a noves situacions. Aquest treball examina el paper del programari lliure en l'àmbit universitari i el seu potencial per a reduir la bretxa digital, presentant també algunes experiències recents.
\end{abstract}

Paraules clau: FOSS, Espai Europeu d'Educació Superior (EEES), Moodle, Open Course Ware (OCW), Gretl

\begin{abstract}
The use of Free Open Source Software (FOSS) provides outstanding advantages in the university context, becoming especially clear in the new framework of the European Higher Education Area (EHEA). The main characteristics of FOSS (free use of programs, access to the source code, study and adaptation, distribution of copies and implementation of improvements that benefit all the community) appear related to competences as creativity, teamwork or adaptation to new situations. This paper examines the role of the free software in the university context, mainly emphasizing its potential to reduce the existing digital divide, and also showing some recent experiences.
\end{abstract}

Key words: Free Open Source Software (FOSS), European Higher Education Area (EHEA), Moodle, Open Course Ware (OCW), Gretl

\footnotetext{
* Proyectos competitivos en cuyo marco se ha desarrollado este trabajo

- Redes internacionales de innovación educativa en Estadística y Econometría, proyecto financiado como Acción 5.3 por el Vicerrectorado de Convergencia Europea, Postgrado y Títulos propios de la Universidad de Oviedo, curso 2006-2007 (Investigadora Principal: Ana Jesús López Menéndez).

- Grupo de Innovación Educativa en Estadística y Econometría, financiado como Acción C en la Convocatoria de Proyectos de Innovación 2006-2007, Vicerrectorado de Calidad e Innovación de la Universidad de Oviedo (Investigadora Principal: Covadonga Caso Pardo).

- Econometría en OCW, Proyecto aprobado en la Convocatoria de Proyectos de Innovación 2010-2011 de la Universidad de Oviedo, Vicerrectorado de In-

formática y Comunicaciones (Investigador Principal: Rigoberto Pérez Suárez).
} 


\section{Introducción}

En el actual contexto de convergencia europea, el software libre puede ser una herramienta estratégica en el ámbito universitario, que aporta ventajas tanto de tipo económico como cultural y tecnológico, facilitando así los objetivos de la declaración de Bolonia (1999) de "establecer una Europa más completa y de mayor alcance construida, en particular, mediante el desarrollo y fortalecimiento de sus dimensiones intelectual, cultural, social, científica y tecnológica".

El software libre se caracteriza por su distribución mediante una licencia que garantiza la libertad de ejecutarla, de conocer el código fuente, de modificarla o mejorarla y de redistribuir copias a otros usuarios. No obstante, dado que - como consecuencia de la ambivalencia del término freeen algunas ocasiones se producen confusiones entre los conceptos software libre y código abierto, en la actualidad se opta a menudo por el término FOSS o FLOSS (Free/Libre Open Source Software) que resulta más amplio e imparcial, al abarcar ambos conceptos. Esta ha sido también nuestra opción en el presente trabajo, cuyo objetivo es analizar el potencial del software libre en el ámbito universitario.

Resulta interesante tener presente que los principales rasgos del software libre aparecen relacionados con algunos de los objetivos del Espacio Europeo de Educación Superior (EEES). Así, tal y como recoge el decálogo de la fundación CENATIC, el uso de software libre conduce a un modelo educativo libre, democrático, sostenible y tecnológicamente competitivo, que por tanto facilitaría la consecución de algunas de las competencias más destacadas en el EEES como la creatividad, la capacidad de aprender, la resolución de problemas o la actualización de conocimientos. Conviene también destacar que el software libre se caracteriza por sus desarrollos centrados en el usuario o cliente, mientras el proceso de convergencia europea ha insistido en la necesidad de situar al estudiante como centro del proceso de enseñanza-aprendizaje.

Además, una coincidencia adicional es que tanto el software libre como el proceso de convergencia europea asignan una importancia destacada al establecimiento de redes y la coopetición como modelo de mejora y evolución.

Como consecuencia de estas reflexiones, este trabajo aborda el papel del software libre como herramienta estratégica en el actual Espacio Europeo de Educación superior, presentando algunas experiencias relativas a su utilización en la enseñanza universitaria tanto presencial como virtual.
Más concretamente, en el apartado que sigue analizamos brevemente la brecha digital y algunos de sus indicadores, describiendo el papel que puede desempeñar en la reducción de esta brecha el software libre, y más concretamente su utilización en educación.

A continuación, en el apartado siguiente describimos nuestras experiencias de implementación de software libre, basadas fundamentalmente en el software econométrico Gretl, el uso de las plataformas Moodle y Open Course Ware (OCW) y las herramientas colaborativas de Google.

\section{Método y análisis de indicadores}

Según la OCDE el término brecha digital hace referencia a las disparidades existentes entre individuos, hogares, empresas y áreas geográficas en el acceso y uso de las Tecnologías de la Información y la Comunicación (TIC). Si bien debido a las limitaciones de información estadística, la brecha más estudiada es la de acceso (a través de indicadores de penetración) cada vez se insiste más en la necesidad de analizar las brechas de uso y de calidad de uso, esta última estrechamente relacionada con aspectos como la seguridad y flexibilidad del software y la capacitación y habilidades de los usuarios. La tabla siguiente resume algunos de los indicadores elaborados por distintos organismos con el objetivo de aproximar la brecha digital en el ámbito internacional y nacional.

Según los datos suministrados por los informes internacionales de CENATIC e ITU, Europa ocupa una posición destacada en acceso y uso de software libre, especialmente en el sector público, situación que previsiblemente se mantendrá con el desarrollo de la Agenda Digital Europea. Además, tal y como cabía esperar, cuando se analiza la situación por países se observa una elevada correlación entre los indicadores de la Sociedad de la Información y del software libre, si bien el caso de España, con una posición intermedia en cuanto a penetración de la Sociedad de la Información, destaca en software libre.

Más concretamente, tal y como muestra la Tabla 2, adoptando como base 100 la media de la Unión Europea, España se sitúa por encima de esta media, con una desviación de tan sólo el 3\% en el índice de Sociedad de la Información, pero en cambio del $48 \%$ en el relativo a software libre. Esta posición favorable es resultado en gran medida del apoyo gubernamental tanto a nivel europeo como nacional y regional, y de hecho, la mayoría de las Comunidades Autónomas presentan iniciativas de software libre, destacando los proyectos de Extremadura y Andalucía.

\begin{tabular}{|l|l|}
\hline INDICADOR (Fuente) & CONTENIDO \\
\hline $\begin{array}{l}\text { Índice de Sociedad de la Información } \\
\text { (CENATIC) }\end{array}$ & $\begin{array}{l}\text { Incluye indicadores de las dimensiones económica, socio-académica, tecnológica y } \\
\text { política. }\end{array}$ \\
\hline Índice de Software Libre (CENATIC) & $\begin{array}{l}\text { Incluye como indicadores de la dimensión socio-académica: participación en la co- } \\
\text { munidad software de fuentes abiertas, formación en software de fuentes abiertas, } \\
\text { grupos de usuarios de Linux, interés por el software de fuentes abiertas, disponibi- } \\
\text { lidad de distribución de GNU/Linux en idioma nativo, disponibilidad de Mozilla en } \\
\text { idioma nativo, nivel de conocimiento de Software de Fuentes Abiertas (SFA) y nú- } \\
\text { mero de usuarios de Linux per cápita. }\end{array}$ \\
\hline ICT Development Index -IDI (ITU) & $\begin{array}{l}\text { Incluye 11 indicadores, de los que 5 son de acceso (peso del 40\%), 3 de uso (40\%) y } \\
\text { 3 de habilidades (20\%). }\end{array}$ \\
\hline Indicadores UNIVERSITIC (CRUE) & $\begin{array}{l}\text { Incluye indicadores de acceso (\% de ordenadores en aulas o de libre disposición que } \\
\text { tienen instalado sistema operativo libre) y de uso (\% de productos utilizados de soft- } \\
\text { ware libre). }\end{array}$ \\
\hline
\end{tabular}

Tabla 1: Algunos indicadores de la brecha digital. Fuente: Elaboración propia a partir de CENATIC, ITU y CRUE. 


\begin{tabular}{|l|l|}
\hline Indice Sociedad de la Información (CENATIC) & 103 \\
\hline Indice Software Libre (CENATIC) & 148 \\
\hline ICT Development Index-IDI (ITU) & 114 \\
\hline $\begin{array}{l}\text { Indicador FOSS en Sector Público } \\
\text { (FLOSSPOLS Survey) }\end{array}$ & 127 \\
\hline
\end{tabular}

Tabla 2: Indicadores de Sociedad de la Información y Software libre en España (UE=100). Fuente: Elaboración propia a partir de CENATIC, ITU y FLOSSPOLS Survey.

Por lo que se refiere al sistema universitario, las ventajas asociadas al software libre aparecen descritas en trabajos como los de Kegel (2003), Bulchand (2005) o Jiménez (2010). Por su parte, el decálogo de razones elaborado por CENATIC para elegir software de fuentes abiertas en educación incluye tanto argumentos de eficiencia económica como otros de tipo educativo, que aparecen estrechamente relacionados con las principales novedades introducidas por el EEES, tal y como resume la Tabla 3.

La información estadística disponible muestra que, si bien todavía queda mucho margen de mejora, las universidades parecen ser conscientes del potencial del software libre. Así, según el último informe UNIVERSITIC (2010) elaborado por la Conferencia de Rectores de las Universidades Españolas (CRUE), la penetración (porcentaje de ordenadores de libre disposición que tienen instalado sistema operativo libre) es del $37 \%$ y, si bien se aprecia una evolución favorable del indicador, la falta de homogeneidad impide cuantificar con exactitud la magnitud de esta mejoría.

También es favorable la evolución de los indicadores de uso, como la proporción de productos de software libre (que actualmente se acerca al 40\%), la utilización de herramientas abiertas en los campus virtuales universitarios (que actualmente representa el 64\% del total, siendo Moodle la plataforma más habitual) y la incorporación de universidades españolas a Open Course Ware (OCW), que supera el $46 \%$.

En el apartado que sigue presentamos algunas experiencias basadas en herramientas de software libre que hemos desarrollado durante los últimos años tanto en la Universidad de Oviedo como en el Campus Virtual Compartido del Grupo G9 (CVC-G9).

\section{Experiencias, resultados y conclusiones}

Cuando ya se han cumplido diez años de la Declaración de Bolonia, nos situamos en una etapa universitaria marcada por la adaptación al Espacio Europeo de Educación Superior (EEES). Tal y como ya hemos señalado, en este contexto resulta destacable el papel desempeñado por el e-learning y el software, herramientas que en nuestra opinión deben ser de acceso libre, flexibles y adaptadas a las características específicas de cada materia y a la correspondiente metodología docente.

De acuerdo con nuestra experiencia, tanto la elección de software como el diseño de la metodología de enseñanzaaprendizaje son claves para el éxito de las experiencias docentes, tanto si éstas son presenciales como de e-learning o blended learning. En los apartados que siguen describimos los principales rasgos del software Gretl y resumimos las experiencias de su implementación en la docencia de las asignaturas Econometría y Predicción Económica y Empresarial.

3.1 El software Gretl

La elección de un software adecuado es un aspecto crucial para el aprendizaje de materias como la Estadística y Econometría. Para llevar a cabo esta elección tenemos en cuenta la adaptación del software a los contenidos de las asignaturas y también su carácter abierto, libre y gratuito, ya que en nuestra opinión las herramientas informáticas no deben incrementar el coste de aprendizaje de nuestros estudiantes.

En el caso de las asignaturas de Econometría, tras llevar a cabo experiencias con otras alternativas como Eviews o SPSS, hemos elegido Gretl por ser un software libre, flexible, amigable y completo, y que se ha revelado como una herramienta muy útil para transmitir y adquirir las competencias ligadas a estas materias.

Gretl, acrónimo de Gnu Regression, Econometrics and Time-series Library, es un paquete de software para análisis econométrico desarrollado en el lenguaje de programación C por A. Cottrell y R.J. Lucchetti. Se trata de software libre y de código abierto que, tal y como recoge su página web (http://gretl.sourceforge.net/) puede ser distribuido y/o modificado bajo los términos de la Licencia Pública General GNU(GPL) según se ha especificado por la Free Software Foundation.

Así pues, la flexibilidad es una de las principales ventajas de Gretl, tanto desde la perspectiva de los profesores como de los estudiantes. De hecho, la estrategia docente con este programa puede ser planteada según distintas posibilidades, desde el trabajo con opciones automáticas de menú hasta el uso de la consola o el desarrollo de programas específicos o funciones de usuario que permiten automatizar Gretl y darle usos avanzados para la investigación.

\begin{tabular}{|l|l|l|}
\hline \multirow{2}{*}{ Razones económicas } & \multicolumn{2}{|l|}{ Razones educativas y su relación con competencias EEES } \\
\hline $\begin{array}{l}\text { Ahorro en implantación, } \\
\text { mantenimiento y licencias }\end{array}$ & $\begin{array}{l}\text { Contribuye a formar personas libres, independien- } \\
\text { tes, críticas y autónomas }\end{array}$ & $\begin{array}{l}\text { Creatividad } \\
\text { Capacidad crítica y autocrítica }\end{array}$ \\
\cline { 2 - 3 } $\begin{array}{l}\text { Independencia de } \\
\text { proveedores }\end{array}$ & $\begin{array}{l}\text { Permite enseñar con herramientas adaptadas a la } \\
\text { realidad del alumnado }\end{array}$ & $\begin{array}{l}\text { Capacidad de aprendizaje, análisis y } \\
\text { razonamiento }\end{array}$ \\
\cline { 2 - 3 } Posibilidad de reutilización & $\begin{array}{l}\text { Facilita que el alumnado disponga en su casa de las } \\
\text { mismas herramientas educativas }\end{array}$ & $\begin{array}{l}\text { Aprendizaje y capacidad para trabajo } \\
\text { autónomo }\end{array}$ \\
\cline { 2 - 3 } Seguridad & Crea una comunidad de conocimiento compartido & Capacidad para trabajo en equipo \\
\cline { 2 - 3 } $\begin{array}{l}\text { Fomento de la innovación } \\
\text { de productos y servicios a } \\
\text { través de empresas locales }\end{array}$ & $\begin{array}{l}\text { Favorece en la persona la libertad de elección } \\
\text { tecnológica }\end{array}$ & $\begin{array}{l}\text { Evoluciona rápidamente y permite una eficaz } \\
\text { solución de problemas }\end{array}$ \\
\hline
\end{tabular}

Tabla 3: Razones para la utilización de software libre abierto (FOSS) en educación. Fuente: Elaboración propia a partir de CENATIC, Comisión Europea y Proyecto TUNING. 


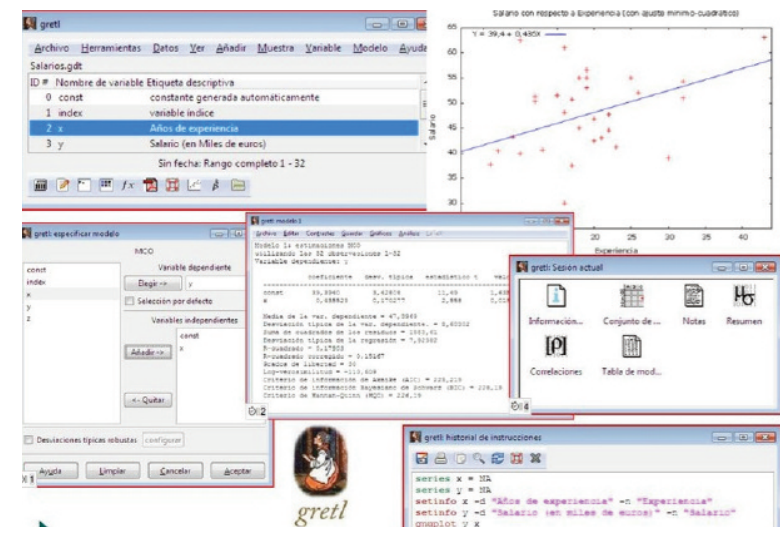

Figura 1: Salidas de pantalla de Gretl

Desde el punto de vista docente, además de las aportaciones técnicas que proporciona Gretl, consideramos interesante destacar dos aspectos que nos parecen fundamentales. El primero de ellos es su enfoque pedagógico, tanto en la presentación de las opciones como en las salidas obtenidas, donde siempre están presentes la hipótesis, el método, la interpretación y la conclusión. Como dice el eslogan de Gretl "es un programa de econometría hecho por económetras", frase a la que podríamos añadir "un programa para la docencia hecho por docentes".

El segundo aspecto básico que destacamos en Gretl es el concepto de sesión. Se trata de un histórico que va registrando nuestra interactividad con Gretl, almacenando en un archivo de sesión las distintas etapas de trabajo realizadas. Esto se complementa además con una opción denominada vista de iconos, donde es posible guardar algunos iconos de actividades concretas (modelos econométricos, gráficos, escalares, etc.) de modo que, si al finalizar el trabajo se guarda la sesión, proporciona un dossier completo del trabajo desarrollado y los hitos principales que aparecen almacenados como iconos.

En definitiva, la gran ventaja de la sesión de Gretl es que permite recuperar el estado de un ejercicio o bien almacenar distintas sesiones en las que hemos estado trabajando con un supuesto. De acuerdo con nuestra experiencia, ésta es una característica valorada positivamente por los estudiantes, tanto en la resolución de supuestos durante las sesiones prácticas con soporte informático como en la realización de trabajos en equipo.

Además, cabe destacar que, como consecuencia de su carácter abierto, Gretl ha desarrollado una comunidad científica que comparte trabajos y experiencias de docencia e investigación mediante listas de distribución, aplicaciones y conferencias bianuales, celebradas hasta ahora en Bilbao (2009) y Torun (2011).

Desde una óptica docente, la implementación de una estrategia combinada al utilizar Gretl permite una mejor asimilación de los contenidos por parte de los estudiantes, que conectan así los resultados proporcionados con el programa con las técnicas estudiadas en las clases expositivas y prácticas. En los apartados que siguen describimos dos experiencias desarrolladas con este programa y referidas a las asignaturas Econometría (troncal del tercer curso de la licenciatura en Economía) y Predicción económica y empresarial (curso online de libre elección impartido en el Campus Virtual Compartido CVC-G9).

3.2 Experiencias con Gretl en Econometría

A lo largo de los últimos años la asignatura Econometría de la licenciatura en Economía se ha ido adaptando progresivamente al método aprender haciendo en cuya implementación hemos tratado de aprovechar el potencial de las Tecnologías de la Información y la Comunicación (TIC) y del software Gretl. Esta metodología resulta especialmente adecuada, teniendo en cuenta que se trata de una materia estrechamente relacionada con las competencias genéricas de análisis, tratamiento e interpretación de la información, consideradas fundamentales en los nuevos grados del ámbito económico.

Por lo que se refiere a las competencias específicas de la asignatura, éstas han sido diseñadas tratando de aproximar los conocimientos, capacidades y destrezas de los estudiantes a los requisitos del mercado laboral, tratando así de atenuar la tradicional distancia existente entre las percepciones de los académicos, los empleadores y los propios estudiantes, que se han puesto de manifiesto en varios estudios incluidos en el proyecto Tuning Educational Structures in Europe.

En esta situación, las competencias genéricas que contemplamos para la asignatura Econometría aparecen estrechamente relacionadas con la metodología docente de la misma, tal y como resumimos en la Tabla 4.

La metodología utlilizada en Econometría se corresponde con el término blended learning, también denominado b-learning o aprendizaje combinado, que según Heinze y Procter (2004) hace referencia al "aprendizaje facilitado a través de la combinación eficiente de diferentes métodos de impartición, modelos de enseñanza y estilos de aprendizaje, y basado en una comunicación transparente de todas las áreas implicadas en el curso". De hecho, aun siendo de carácter presencial, esta asignatura se incorporó hace ya más de una década a AulaNet, campus virtual de la Universidad de Oviedo, con un doble objetivo: poner a disposición de los estudiantes una amplia variedad de materiales docentes y proporcionarles un amplio abanico de canales de comunicación online (correo electrónico, foros, wikis, etc.).

\begin{tabular}{|c|c|}
\hline $\begin{array}{l}\text { Métodos de ense- } \\
\text { ñanza aprendizaje }\end{array}$ & Competencias \\
\hline Sesiones teóricas & $\begin{array}{l}\text { Conocimientos generales básicos } \\
\text { Capacidad de análisis y síntesis } \\
\text { Capacidad de aprendizaje }\end{array}$ \\
\hline Sesiones prácticas & $\begin{array}{c}\text { Capacidad de aplicar la teoría a la } \\
\text { práctica } \\
\text { Resolución de problemas } \\
\text { Conocimiento de segundo idioma }\end{array}$ \\
\hline $\begin{array}{c}\text { Trabajo en equipo } \\
\text { con Gretl } \\
\text { Construcción de } \\
\text { base de datos, } \\
\text { especificación y } \\
\text { estimación de } \\
\text { modelos, } \\
\text { contrastes, análisis, } \\
\text { predicción, } \\
\text { exposición oral, } \\
\text { informe final }\end{array}$ & $\begin{array}{c}\text { Trabajo en equipo } \\
\text { Habilidades de manejo de } \\
\text { información } \\
\text { Creatividad } \\
\text { Adopción de decisiones } \\
\text { Adaptabilidad a nuevas situaciones } \\
\text { Comunicación oral y escrita } \\
\text { Atención a la calidad } \\
\text { Habilidades de investigación }\end{array}$ \\
\hline $\begin{array}{l}\text { Seguimiento y au- } \\
\text { toevaluaciones } \\
\text { Encuestas online }\end{array}$ & $\begin{array}{c}\text { Habilidad de trabajar } \\
\text { autónomamente } \\
\text { Capacidad crítica y autocrítica } \\
\text { Compromiso ético }\end{array}$ \\
\hline
\end{tabular}

Tabla 4: Metodología docente y competencias de Econometría. Fuente: Elaboración propia. 


\begin{tabular}{|l|l|l|}
\hline Secciones & Aspectos cuantitativos & Aspectos cualitativos \\
\hline Trabajo Personal & Horas de estudio & Nivel de dificultad percibida \\
\hline Trabajo en Equipo & $\begin{array}{l}\text { Horas dedicadas a base de datos, estima- } \\
\text { ción de modelos, contraste de hipótesis, } \\
\text { predicción, exposición oral, informe }\end{array}$ & $\begin{array}{l}\text { Dificultad percibida. Satisfacción con Gretl. Percepción } \\
\text { de esfuerzo personal y del equipo. Valoración de la cali- } \\
\text { dad del trabajo y comparación con otros equipos }\end{array}$ \\
\hline Evaluación continua & & $\begin{array}{l}\text { Dificultad percibida de las cuestiones de seguimiento. } \\
\text { Satisfacción con el sistema de evaluación }\end{array}$ \\
\hline Visión General & $\begin{array}{l}\text { Nivel de satisfacción con la asignatura. Opinión sobre ad- } \\
\text { quisición de competencias. Comentarios y sugerencias }\end{array}$ \\
\hline
\end{tabular}

Tabla 5: Encuestas online realizadas a los estudiantes de Econometría

En lo que se refiere al primer objetivo, los materiales docentes incorporados al campus virtual han ido evolucionando, adaptándose a la plataforma universitaria utilizada (inicialmente AulaNet, posteriormente basada en WebCT y actualmente en Moodle) y tratando de garantizar la máxima compatibilidad de los archivos.

En la actualidad esta plataforma se combina con el uso de Google Docs como una herramienta básica para la elaboración de los contenidos de la asignatura, que en nuestra opinión aporta tres beneficios claros. En primer lugar, garantiza la estandarización de formatos, solucionando así los problemas detectados hace algunos años cuando los estudiantes utilizaban distintos sistemas operativos (Windows, Mac, Linux) o distintos programas (OpenOffice, MsOffice 2003, MsOffice 2007, etc.), que condicionaban la visualización de las presentaciones, detectándose errores en las formas y en las fórmulas.

En segundo lugar Google Docs facilita a los profesores el trabajo colaborativo y la actualización continua de los contenidos (que aparecen embebidos en la página de la asignatura, de modo que los estudiantes disponen de todo el material centralizado en el campus virtual y visualizan la última versión de cada documento). Además, en tercer lugar, pero no menos importante, de este modo les transmitimos a los estudiantes una manera fácil y gratuita de trabajar colaborativamente, que según nuestra experiencia les resulta muy útil para la realización de los trabajos en equipo, abriendo también nuevas posibilidades de colaboración para el futuro.

Cabe además señalar que la difusión de los contenidos de Econometría ha experimentado un salto cualitativo recientemente, con su incorporación al portal OCW de la Universidad de Oviedo, que se incluye en la iniciativa Open Course Ware para promover el acceso libre y sin restricciones al conocimiento, puesta en marcha en 2001 por el Massachussets Institute of Technology (MIT), e impulsada en España en el marco de OCW-Universia a través de su portal universitario.

Por lo que se refiere a la comunicación, que consideramos parte fundamental del proceso de enseñanza-aprendizaje, se combina el uso del correo electrónico (para consultas puntuales) con los foros para asuntos de interés más general (noticias, software Gretl, y trabajos en equipo, habilitándose en este caso foros a los que pertenecen los 3 ó 4 estudiantes integrantes de cada equipo y los profesores).

Además, se utilizan de forma complementaria otros recursos como los wikis, a través de los cuales los estudiantes pueden elaborar documentos colaborativos, y que facilitan también la organización del calendario de exposiciones de trabajos y la realización de debates.
A las dos funciones ya descritas para el campus virtual (suministrar materiales docentes y activar canales de comunicación) se añade a partir del año 2004 otra adicional, al iniciarse en la asignatura Econometría las experiencias piloto en el contexto del proceso de convergencia europea, en las que resultaba crucial contar con la participación de los estudiantes. Así, el campus virtual nos permite recabar a través de encuestas online las valoraciones de los estudiantes sobre distintos aspectos del proceso de enseñanzaaprendizaje, como su dedicación en horas a la asignatura y su percepción sobre dificultad y nivel de satisfacción con los distintos elementos del proceso docente.

Tal y como cabía esperar, de todos los aspectos investigados en la encuesta la valoración del trabajo personal en horas es la que encierra una mayor dificultad para los estudiantes. No obstante, esta información, que se solicitaba inicialmente con el objetivo de estimar los créditos ECTS de la asignatura, se mantiene en la actualidad para analizar la estabilidad de las conclusiones.

En términos generales, los resultados de las encuestas online de Econometría, que corresponden a las respuestas facilitadas por 142 estudiantes, muestran una elevada dispersión en las respuestas sobre trabajo personal, reflejando tanto la dificultad de cálculo como la heterogeneidad existente entre los estudiantes, rasgo estable a lo largo de los años y que en nuestra opinión debe ser tenido en cuenta en el diseño de la estrategia docente.

Frente a esta diversidad en los tiempos de trabajo personal, se aprecia una considerable homogeneidad en los niveles de dificultad percibidos para las actividades docentes y en la opinión sobre los criterios de evaluación, que como resume la Tabla 6 resulta muy favorable.

En cuanto a la percepción de las competencias adquiridas, en la Figura 2 se observa que los estudiantes valoran especialmente el software Gretl, cuyo manejo hemos tratado de facilitar a través de ayudas en el campus virtual, inclu-

\begin{tabular}{|l|c|c|}
\hline & $\begin{array}{c}\text { Nivel de } \\
\text { dificultad } \\
\text { (\% de respues- } \\
\text { tas "Difícil" o } \\
\text { "Muy difícil") }\end{array}$ & $\begin{array}{c}\text { Nivel de } \\
\text { acuerdo (\% de } \\
\text { respuestas } \\
\text { "Acuerdo" o } \\
\text { "Acuerdo } \\
\text { total") }\end{array}$ \\
\hline Prácticas con Gretl & $10,5 \%$ & $70,0 \%$ \\
Trabajo en equipo & $73,3 \%$ & $94,1 \%$ \\
Evaluación continua & $17,9 \%$ & $70,8 \%$ \\
Examen final & $8,3 \%$ & $100 \%$ \\
\hline
\end{tabular}

Tabla 6: Principales resultados de las encuestas online de Econometría. 


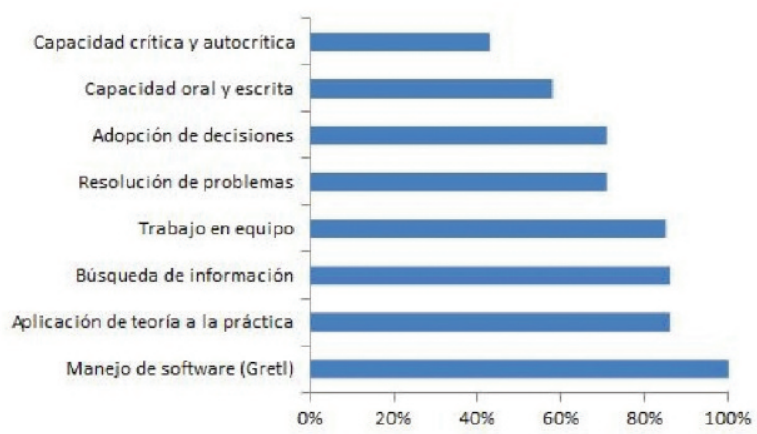

Figura 2: Valoración de los estudiantes de las competencias de Econometría. Fuente: Elaboración propia.

yendo -además del Manual de usuario- una guía denominada Manos a la obra, un foro específico sobre Gretl y una sección FAQ (Frequently Asqued Questions).

También reciben buena valoración la capacidad de aplicar los conocimientos teóricos a la práctica, las habilidades de gestión de la información y el trabajo en equipo, que constituye una preocupación fundamental del alumnado, y se cita como fuente de problemas y conflictos, asociados a los desequilibrios en la organización y reparto de tareas.

Por lo que se refiere a la baja valoración de la capacidad crítica y autocrítica, los resultados de las encuestas coinciden con nuestra percepción, ya que existen todavía importantes reticencias a la hora de manifestar -tanto en directo como a través del foro u otro medio- la opinión respecto al trabajo de los compañeros, posiblemente debido a que esta práctica está todavía poco asentada en nuestro sistema universitario.

Cabe por último señalar que, con el objetivo de garantizar la coherencia del proceso docente, la metodología descrita para la asignatura Econometría lleva asociado un sistema de evaluación continua, que asigna un peso del 30\% al trabajo en equipo con Gretl, un 20\% a las cuestiones de seguimiento mensuales y el 50\% restante al examen final. La aplicación de este sistema ha permitido mejorar tanto los indicadores de rendimiento académico de la asignatura como la valoración de los estudiantes en la Encuesta General de Enseñanza.

La Figura 3 recoge la tasa de éxito (proporción de estudiantes que superan la asignatura sobre el total de matriculados) que es superior a la media del departamento, del campus y del conjunto de la Universidad.

3.3 Experiencias con Gretl en un Campus Virtual: Predicción económica y empresarial

Las experiencias docentes relativas a la asignatura Predicción económica y empresarial presentan algunas analogías con las vistas anteriormente para Econometría, si bien el principal rasgo distintivo de esta asignatura es que su docencia se realiza totalmente online, en el marco del Campus Virtual Compartido CVC-G9, por lo que resulta especialmente importante el carácter abierto de la plataforma virtual (en este caso Moodle). Además, en el contexto del Espacio Europeo de Educación Superior, este tipo de experiencias supone un impulso a la movilidad virtual y el trabajo colaborativo entre universidades, contribuyendo así a atenuar la brecha digital existente entre ellas.

En lo que se refiere a sus contenidos, esta materia trata de proporcionar herramientas para anticipar el comportamiento de las principales magnitudes económicas y su planteamiento es eminentemente práctico y centrado en el

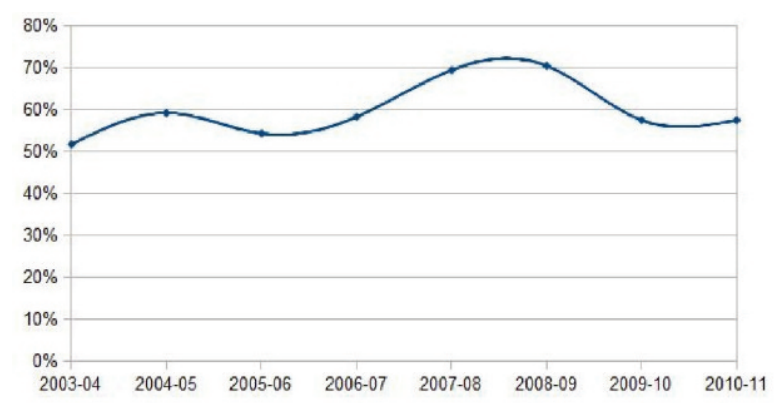

Figura 3: Evolución de la tasa de éxito de Econometría. Fuente: Elaboración propia.

estudiante, mientras los profesores-tutores actuamos como facilitadores del aprendizaje.

Durante los últimos cinco cursos académicos la docencia de esta asignatura se ha desarrollado en el marco del Campus Virtual Compartido CVC-G9, involucrando a un buen número de estudiantes procedentes de nueve universidades distintas (Universidad de Cantabria, Universidad de Castilla-La Mancha, Universidad de Extremadura, Universidad de Illes Balears, Universidad de La Rioja, Universidad Pública de Navarra, Universidad del País Vasco, Universidad de Oviedo y Universidad de Zaragoza) y de una amplia variedad de titulaciones, dado su carácter de materia de libre elección. En este tiempo hemos prestado especial atención a la elaboración de materiales docentes de calidad, la elección del software Gretl y las herramientas de comunicación online, factor clave para tratar de reducir en la medida de lo posible la tasa de abandono, que es uno de los mayores riesgos de la enseñanza no presencial. Afortunadamente, los resultados obtenidos pueden considerarse satisfactorios, teniendo en cuenta el número de estudiantes matriculados, los indicadores académicos y la valoración en las encuestas.

El papel de Gretl en la asignatura Predicción económica y empresarial resulta especialmente destacable ya que, debido a su menor contenido teórico, el uso de este software facilita la comprensión de técnicas y la resolución de problemas. El proceso docente se apoya también en las tutorías online, que permiten plantear dudas a los profesores- tutores, y los foros que proporcionan una comunicación más rica en la que interactúan los estudiantes. Además, con carácter complementario se convocan varias sesiones chat a lo largo del cuatrimestre, proporcionando así una herramienta de comunicación instantánea.

Según la información disponible, los estudiantes asignan una gran valoración a estas herramientas de comunicación y también al acceso a información personalizada. De ahí que, a lo largo del cuatrimestre, se vayan incorporando a la plataforma los resultados de las pruebas de evaluación continua (de acceso restringido a cada estudiante), junto a algunas estadísticas, resumen y comentarios sobre los errores más frecuentes.

El sistema de evaluación de la asignatura se adapta a la metodología descrita, y así la calificación final se obtiene como resultado de los siguientes criterios:

- Calificaciones de las autoevaluaciones (40\%)

- Calificaciones de los cuestionarios sobre Gretl (40\%)

- Participación online (20\%)

Este método de evaluación conduce a resultados satisfactorios, ya que desde su inicio han ido reduciéndose gradualmente las tasas de abandono y aumentando las de éxito. 


\begin{tabular}{|c|c|c|c|c|}
\hline Cuestión & \multicolumn{4}{|c|}{ Respuestas } \\
\hline $\begin{array}{c}\text { Razones para ele- } \\
\text { gir una asignatura }\end{array}$ & $\begin{array}{c}\text { Consejos de } \\
\text { compañeros (42\%) }\end{array}$ & $\begin{array}{c}\text { Web universitaria } \\
(30 \%)\end{array}$ & $\begin{array}{c}\text { Información en } \\
\text { campus (6\%) }\end{array}$ & Otros (22\%) \\
\hline Lugar de estudio & Trabajo (3\%) & Campus (16\%) & Hogar (78\%) & Cibercafé (2\%) \\
\hline $\begin{array}{c}\text { Frecuencia de } \\
\text { acceso }\end{array}$ & Semanal (35\%) & $\begin{array}{c}\text { Dos veces por semana } \\
(29 \%)\end{array}$ & $\begin{array}{c}\text { Tres o más veces por } \\
\text { semana (20\%) }\end{array}$ & $\begin{array}{c}\text { Menos de una vez por } \\
\text { semana (16\%) }\end{array}$ \\
\hline $\begin{array}{c}\text { Nivel de dificultad } \\
\text { y esfuerzo }\end{array}$ & $\begin{array}{c}\text { Similar a otras } \\
\text { materias de libre } \\
\text { elección (45\%) }\end{array}$ & $\begin{array}{c}\text { Superior a otras } \\
\text { materias de libre } \\
\text { elección (45\%) }\end{array}$ & $\begin{array}{c}\text { Inferior a otras } \\
\text { materias de libre } \\
\text { elección (10\%) }\end{array}$ & \\
\hline
\end{tabular}

Tabla 7: Algunos resultados de la encuesta online de Predicción económica y empresarial. Fuente: Elaboración propia.

Por lo que se refiere a la percepción de los estudiantes que cursan la materia, las encuestas online realizadas anualmente a través del campus virtual permiten conocer tanto el nivel de dificultad percibido como el nivel de satisfacción con la experiencia, las competencias adquiridas y las distintas herramientas utilizadas en el proceso.

Si bien la tasa de respuesta no es tan elevada como nos gustaría (en torno al 50\%), los resultados obtenidos muestran algunos hechos interesantes. Así, aunque existe una considerable heterogeneidad de estudiantes, que pertenecen a distintas universidades, titulaciones y cursos, las respuestas que proporcionan son bastante homogéneas e indican que los estudiantes suelen elegir esta materia siguiendo consejos de compañeros que la habían cursado previamente, y acceden al campus virtual una o dos veces por semana, generalmente desde sus domicilios. Además, tal y como recoge la Tabla 7, los estudiantes consideran que el nivel de dificultad de Predicción económica y empresarial y el esfuerzo requerido para superarla son similares o superiores a los de otras materias de libre elección.

\section{Reflexiones finales}

A pesar de los avances observados durante los últimos años en la incorporación de software libre al ámbito universitario, queda todavía un amplio margen de mejora, tanto en lo que se refiere a los indicadores de acceso y uso como a la calidad. Así lo confirman las iniciativas educativas realizadas con éxito en distintas regiones (GNU/Linex-Extremadura, Guadalinex-Andalucía, Linkat- Cataluña, Molinux-Castilla La Mancha, MAX-Madrid, etc.) y también diversas experiencias universitarias que muestran las ventajas de aplicaciones como Gretl o R en la docencia de asignaturas de Estadística y Econometría y su potencial para la adquisición de competencias.

En nuestro caso, las experiencias en las asignaturas de Econometría y Predicción económica y empresarial permiten extraer algunas conclusiones y reflexiones de interés para la implementación de los nuevos grados en el marco del Espacio Europeo de Educación Superior.

Si bien el desarrollo de estas experiencias exige un considerable esfuerzo por parte de todos los agentes implicados, hemos constatado el gran potencial que tienen las herramientas de $e$-learning, blended learning y el software Gretl en el proceso docente.

Así, el campus virtual permite gestionar una amplia variedad de materiales docentes y además proporciona herramientas de comunicación de gran utilidad en el proceso enseñanza-aprendizaje. Por su parte, el programa Gretl se ha revelado, gracias a su carácter libre, flexibilidad y capacidad didáctica, como muy adecuado para el aprendizaje de la Econometría y las técnicas de predicción.
Las encuestas online, implementadas en el campus virtual para las dos asignaturas descritas, nos permiten acceder a la información proporcionada por los estudiantes sobre tiempos de trabajo, dificultad percibida y nivel de consecución de las competencias y muestran, en ambos casos, resultados razonablemente satisfactorios, identificando también algunos puntos débiles para los que deberemos diseñar acciones de mejora. En definitiva, cuando ya hemos superado el umbral del año 2010, adoptado como referencia en el proceso de convergencia europea, las experiencias descritas en este trabajo nos animan a seguir avanzando en esta dirección, convencidos de que el esfuerzo merece la pena y de que durante el proceso también nosotros hemos conseguido aprender haciendo.

Por lo que se refiere al futuro, según M. Shuttleworth de Ubuntu, se espera que las distribuciones de software serán universales pero con personalizaciones culturales, sectoriales y profesionales, que pueden resultar de gran interés en el ámbito universitario. Por otra parte las iniciativas como oficinas de software libre, declaraciones institucionales, distribuciones propias, programas de postgrado sobre FOSS y licencias Creative Commons deben afrontar todavía obstáculos como el desconocimiento, la resistencia al cambio y la insuficiente colaboración.

En definitiva, la contribución del software libre en el ámbito universitario puede traducirse en un doble efecto: aumento de la eficiencia económica y reducción de la brecha digital, y por tanto supone una evolución favorable de los dos componentes del bienestar (aumento del crecimiento y reducción de la desigualdad). No obstante, conviene tener presente que, si bien en este trabajo nos hemos centrado en los efectos positivos del FOSS sobre el sistema universitario, es de esperar que se produzca también causalidad en el otro sentido (contribución universitaria al desarrollo del FOSS). De ahí la importancia de fomentar la colaboración de las universidades con el sector público, el sector privado y las comunidades de desarrolladores, que forman también parte del denominado ecosistema del software libre.

\section{Bibliografía}

Bulchand, Jacques (2005). Software libre en la Universidad. En Libro Blanco del Software Libre en España (II), A. Abella y M.A. Segovia Editores.

http://www.libroblanco.com/document/II_libroblanco_del_software_libre.pdf, 10 de Mayo de 2011.

CENATIC (2009). Estudio sobre la situación actual del Software de Fuentes Abiertas en las Universidades y Centros $I+D$ españoles.

http://observatorio.cenatic.es/phocadownload/inform es/informe_universidad.pdf 
CENATIC (2010). Informe sobre el panorama internacional del Software de Fuentes Abiertas 2010. Badajoz. www.cenatic.es/publicaciones/category/1?download $=33$

Comisión Europea (2001), Plan de acción eLearning. Concebir la educación del futuro, Comunicación de la Comisión al Consejo y al Parlamento Europeo.

Cottrell, Allin; Lucchetti, Ricardo Jack (2008). Gretl User's Guide, Gnu Rregression, Econometrics and Time Series, http://gretl.sourceforge.net/

European Commission (2009). Bologna beyond 2010. Report on the development of the European Higher Education Area, Background paper for the Bologna Follow-up Group prepared by the Benelux Bologna Secretariat, Louvain-la-Neuve Ministerial Conference, 28-29 April.

González, Julia; Wagenaar, Robert (2003). Tuning Educational Structures in Europe,

http://www.relint.deusto.es/TUNINGProject/spanish /doc fase1/Tuning\%20Educational.pdf

González, Julia; Wagenaar, Robert (2005). Tuning Educational Structures in Europe II. Universities' contribution to the Bologna Process, http://www.tuning.unideusto.org/tuningeu/

Ghosh, Rishab A. (2005). An Economic Basis for Open Standards.

http://www.intgovforum.org/Substantive_1st_IGF/op enstandards-IGF.pdf, 6 de Mayo de 2011

Heinze, Aleksej; Procter, Chis (2004). "Reflections on the Use of Blended Learning”. Education in a Changing Environment conference proceedings, University of Salford, Salford, Education Development Unit http://www.ece.salford.ac.uk/proceedings/papers/ah 04.rtf

ITU (2011). Measuring the WSIS Targets. Ginebra. International Telecommunication Union.

ITU (2010). Measuring the Information Society. Geneve.

Jiménez, Tomás (2010). Software Libre. En CRUE (Ed.), UNIVERSITIC 2010: Evolución de las TIC en el Sistema Universitario Español 20o6-2010, Madrid: CREU, pp. 113-115

Kegel, Dan (2003). The Case for Linux in Universities. http://www.kegel.com/linux/edu/,10 de Mayo de 2011.
López, Ana Jesús; Pérez, Rigoberto (2009). "Towards the European Higher Education Area. Blended learning experiences in Econometrics", V International Conference on Multimedia and Communication Technologies in Education m-ICTE 2009, Lisboa, 22-24 de Abril, http://www.formatex.org/micte2009/volume2.htm.

López, Ana Jesús; Pérez, Rigoberto; Mayor, Matías (2006). "Experiencias de enseñanza y aprendizaje en el Espacio Europeo de Educación Superior. El caso de la Econometría”, Congreso Internacional de Docencia Universitaria e Innovación CIDUI 2006, Barcelona, 57 de Julio.

Pérez, Rigoberto; López, Ana Jesús (2009).“Teaching and Learning Econometrics with Gretl. Summarizing some experiences" en Econometrics with Gretl. Proceedings of the Gretl Conference 2009, Bilbao: Universidad del País Vasco, pp.191-201. .

Pérez, Rigoberto; López, Ana Jesús (2011)."El uso del software libre en el EEES: abriendo mentes, cerrando brechas" en Evaluación de la Calidad de la Educación Superior y de la Investigación (VIII Foro), Santander.

The European Students' Union (2009). Bologna With Student Eyes 2009, Project "Enhancing the Student Contribution to Bologna Implementation" (ESCBI), European Commission.

Wheeler, David A. (2007). Why Open Source Software/Free Software (OSS/FS, FLOSS or FOSS)? Look at the Numbers!, http://www.dwheeler.com/oss_fs_why.html, $18 \mathrm{de}$ Mayo de 2011. 